\title{
Fuzzy image inpainting aimed to medical images
}

\author{
Pavel Vlašánek \\ Institute for Research \\ and Applications of Fuzzy \\ Modeling \\ 30. dubna 22 \\ 70103 Ostrava 1, Czech \\ Republic \\ pavel.vlasanek@osu.cz
}

\begin{abstract}
This paper focuses on a reconstruction of low color depth images using fuzzy mathematics. For demonstration purposes, we chose medical images taken from magnetic resonance imaging (MRI) and computed tomography (CT). The proposed technique is based on idea of diffusion where pixels surrounding damaged region are used to determine the corrupted ones. As it is illustrated, the classical diffusion techniques are not so effective. In the paper, we describe the reason why and propose the solution in a form of the new algorithm. The algorithm is demonstrated and visually compared with another ones.
\end{abstract}

\section{Keywords}

image inpainting, fuzzy transform, clustering

\section{INTRODUCTION}

Image inpainting based on fuzzy mathematics is topic which belongs to soft computing image processing. The term stands for image processing tasks performed by soft computing techniques such as neural networks or fuzzy based approaches. The image inpainting stands for unwanted region removal followed by its recovery.

The image inpainting was introduced by Bertalmio et al. [1], who proposed to use partial differential equations (PDE). It consists in a propagation of the colors inward damaged area. The technique is successful for corruptions like scratches or thin inscriptions. In general, small damaged regions without any big hole. The diffusion based inpainting applied to the big hole leads to the unnaturally blurry reconstruction because of its inability to keep the patterns. The solution for this problem is in the patch based approach [2, 3]. For this approach, the algorithm searches for a square patches used latter as a replacement for the similar patches in the damaged region. This approach keeps the potential patterns and/or textures. From techniques using both, we can mention $[4,5]$.

Permission to make digital or hard copies of all or part of this work for personal or classroom use is granted without fee provided that copies are not made or distributed for profit or commercial advantage and that copies bear this notice and the full citation on the first page. To copy otherwise, or republish, to post on servers or to redistribute to lists, requires prior specific permission and/or a fee.
As we stated above, the problem of diffusion is in blurring. The reason is in isotropic nature of the common algorithms which propagates the information to the all directions. We propose to segment the input image first, and apply the inpainting on the each segment separately. The algorithm is demonstrated on the low color depth images where successfully prevents creation of the unwanted artifacts.

Structure of this paper is as follows. Notation and history of image inpanting is given in Section 2. Section 3 contains information about fuzzy transform and fuzzy clustering. The details of the proposed algorithm are described in Section 4. Section 5 includes experiments and comparison. The paper is concluded in Section 6.

\section{NOTATION AND HISTORY}

Notation used in the paper is as follows. Discrete image $I$ is a $2 \mathrm{D}$ matrix function such as $I$ : $[0, M]_{Z} \times[0, N]_{Z} \rightarrow[0,255]_{Z}^{3}$, where $[0,255]_{Z}^{3}$ stands for the pixel intensities in three color channels. We denote $[0, M]_{Z}=\{0,1,2, \ldots, M\}, \quad[0, N]_{Z}=\{0,1,2, \ldots, N\}$ and $[0,255]_{Z}=\{0,1,2, \ldots, 255\}$. Thus, image width is equal to $M+1$ and height to $N+1$. Image $I$ is partially known. The region $\Phi$ stands for known (undamaged) pixels and $\Omega$ for unknown (undefined, damaged). Their border is denoted by $\delta \Omega$ and taken as unknown. We assume that $\Phi \cap \Omega=\emptyset$ and $\Phi \cup \Omega \cup \delta \Omega=[0, M]_{Z} \times[0, N]_{Z}$. To distinguish between $\Phi$ and $\Omega$, a mask $S$ is used. The mask is a binary image where black pixels denote unknown area $\Omega \cup \delta \Omega$. Let us remark that the mask must be given by user. 
The digital image inpainting is demonstrated in Fig. 1.

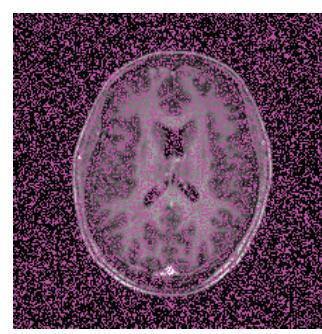

(a) Input image $I$

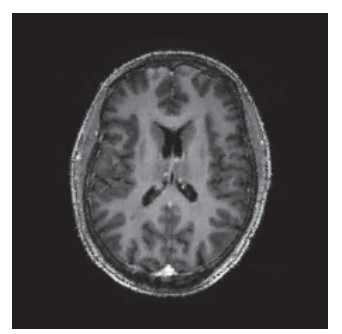

(b) Reconstructed image $O$
Figure 1: Demonstration of image inpainting. The noise was erased by proposed algorithm.

The general principle and formal description was given by pioneers Bertalmio et al. [1] and it is as follows

$$
I^{z+1}(x, y)=I^{z}(x, y)+\Delta t I_{t}^{z}(x, y), \forall(x, y) \in \Omega,
$$

where $z$ is the iteration step, $(x, y)$ stands for pixel coordinates, $\Delta t$ is the rate of improvement and $I_{t}^{z}(x, y)$ stands for the update of image $I^{z}(x, y)$. The $I_{t}^{z}(x, y)$ step is computed using a smoothness $L^{z}(x, y)$ estimated by Laplacian operator. The change of smoothness is propagated from known region $\Phi$ to the border $\delta \Omega$ in the direction $\vec{N}(x, y)$. This statement is shown in Fig. 2 .

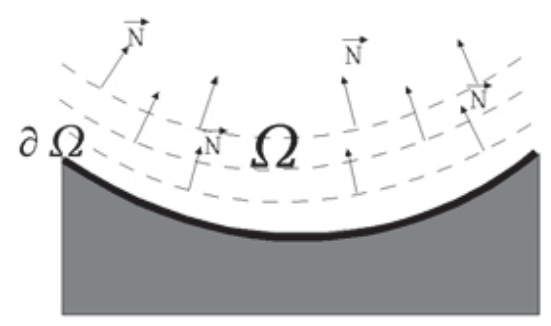

Figure 2: Illustration of the Bertalmio et. al. inpainting process. (Figure taken from [1])

Bertalmio et al. [1] proposed formula

$$
I_{t}^{z}(x, y)=\overrightarrow{\delta L^{z}}(x, y) \cdot \vec{N}^{z}(x, y)
$$

where $\overrightarrow{\delta L} z(x, y)$ is the measure of the change in smoothness $L^{z}(x, y)$ and direction defined as orthogonal to image gradient

$$
\vec{N}^{z}(x, y)=\left(\nabla I^{z}(x, y)\right)^{\perp} .
$$

Another principle was proposed by Ogden et al. [6]. The authors used Gaussian pyramid based technique. The technique builds the pyramid using convolution and sub-sampling followed by linear interpolation.
Elad et al. [7] recommended to separate image to the geometry part $D_{g}$ and texture part $D_{t}$ where inpainting is done separately for both. Image decomposition using $D_{g}$ and $D_{t}$ matrices is as follows

$$
I=D_{g} \alpha_{g}+D_{t} \alpha_{t},
$$

where $\alpha_{g}$ and $\alpha_{t}$ stands for geometry and texture coefficients. Its illustration is in Fig 3.

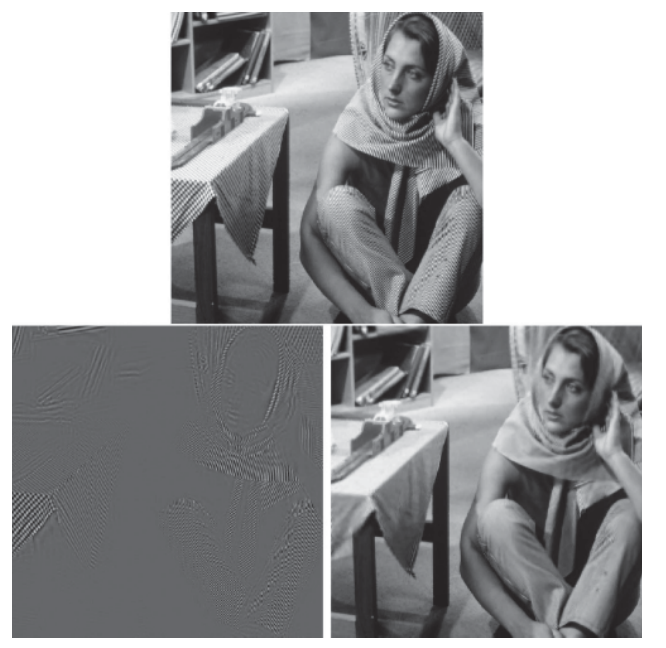

Figure 3: Image decomposed to the texture (bottom left) and geometry (bottom right) parts (figure taken from [7])

Their image representation is defined as follows

$$
\min _{\left(\alpha_{g}, \alpha_{t}\right): I=D_{g} \alpha_{g}+D_{t} \alpha_{t}}\left\|\alpha_{g}\right\|_{p}+\left\|\alpha_{t}\right\|_{p}
$$

where $p$ is the coefficient of the $\ell$-norm $\|\alpha\|_{p}=\left(\sum\|\alpha(g)\|^{p}\right)^{1 / p}$. The model proposed by Elad et al. [7] is as follows

$$
\min _{\left(\alpha_{g}, \alpha_{t}\right)}\left\|\alpha_{g}\right\|_{1}+\left\|\alpha_{t}\right\|_{1}+\lambda\left\|I-D_{g} \alpha_{g}-D_{t} \alpha_{t}\right\|_{2}^{2}+\gamma T V\left(D_{g} \alpha_{g}\right) .
$$

TV stands for total variation, $p=1$ and $\lambda, \gamma>0$. Adaptation for image inpainting is

$$
\min _{\left(\alpha_{g}, \alpha_{t}\right)}\left\|\alpha_{g}\right\|_{1}+\left\|\alpha_{t}\right\|_{1}+\lambda\left\|C\left(I-D_{g} \alpha_{g}-D_{t} \alpha_{t}\right)\right\|_{2}^{2}+\gamma T V\left(D_{g} \alpha_{g}\right),
$$

where $C=1$ stands for the undamaged pixels and $C=0$ for damaged ones. More information is in [7]. Our novel algorithm extends the idea of the separation and combines it with diffusion approach using fuzzy mathematics.

For sake of comparison, let us mention two other techniques ${ }^{1}$. First of them is based on Navier-Stokes equation [8]. The authors use physics of viscous fluid motion to propagate the information inward damaged region. Second one is based on fast marching method

${ }^{1}$ Both of them are implemented in OpenCV framework. 
(FMM) [9] which highlights an importance of filling order.

\section{PRELIMINARIES}

First to describe is F-transform which is a technique for changing an image representation. Second one is clustering algorithm fuzzy C-Means.

\subsection{F-transform}

We propose a fuzzy based approach to solve image inpainting task [10]. Let us describe fuzzy transform (Ftransform) [11] given by a fuzzy partition satisfying following definition. Fuzzy sets $A_{0}, \ldots, A_{m} ; m<M$ identified with their membership functions (basic functions) $A_{0}, \ldots, A_{m}:[0, M] \rightarrow[0,1]$, establish a fuzzy partition of $[0, M]$ with nodes $0=x_{0}<x_{1}<\cdots<x_{m}=M$ if the following conditions are fulfilled:

1) $A_{k}:[0, M] \rightarrow[0,1], A_{k}\left(x_{k}\right)=1$;

2) $A_{k}(x)=0$ if $x \notin\left(x_{k-1}, x_{k+1}\right), k=0, \ldots, m$;

3) $A_{k}(x)$ is continuous;

4) $A_{k}(x)$ strictly increases on $\left[x_{k-1}, x_{k}\right]$, $k=2, \ldots, m$; and strictly decreases on $\left[x_{k}, x_{k+1}\right], k=$ $1, \ldots, m-1$

5) $\sum_{k=0}^{m} A_{k}(x)=1, x \in[0, M]$.

Assume that fuzzy sets $A_{0}, \ldots, A_{m}$ establish a fuzzy partition of $[0, M]$. The vector of real numbers $\mathbf{F}_{m}[I]=$ $\left(F_{0}, \ldots, F_{m}\right)$ is the (direct) discrete $F$-transform of $I$ w.r.t. $A_{0}, \ldots, A_{m}$ where the component $F_{k}$ is defined by

$$
F_{k}=\frac{\sum_{x=0}^{M} A_{k}(x) I(x)}{\sum_{x=0}^{M} A_{k}(x)}, k=0, \ldots, m .
$$

Let us introduce F-transform of a 2D gray-scale image $I$. Let $A_{0}, \ldots, A_{m}$ and $B_{0}, \ldots, B_{n}$ be basic functions, $A_{0}, \ldots, A_{m}:[0, M] \rightarrow[0,1]$ be fuzzy partition of $[0, M]$ and $B_{0}, \ldots, B_{n}:[0, N] \rightarrow[0,1]$ be fuzzy partition of $[0, N]$. If for all $k \in 0, \ldots, m(\exists x \in[0, M]) A_{k}(x)>0$, and for all $l \in 0, \ldots, n(\exists y \in[0, N]) B_{l}(y)>0$ with respect to $\Phi$, we say that the set of pixels $\Phi$ is sufficiently dense with respect to the chosen partitions.

We say that the $m \times n$-matrix of real numbers $\left[F_{k l}\right]$ is called the (discrete) F-transform of $I$ with respect to $\left\{A_{0}, \ldots, A_{m}\right\}$ and $\left\{B_{0}, \ldots, B_{n}\right\}$ if for all $k=0, \ldots, m, l=0, \ldots, n$,

$$
F_{k l}=\frac{\sum_{y=0}^{N} \sum_{x=0}^{M} I(x, y) A_{k}(x) B_{l}(y)}{\sum_{y=0}^{N} \sum_{x=0}^{M} A_{k}(x) B_{l}(y)} .
$$

The coefficients $F_{k l}$ are called components of the $F$ transform. The formula (7) is called direct step. In order to reconstruct the original function, it is usually followed by inverse step as follows

$$
O(x, y)=\sum_{k=0}^{m} \sum_{l=0}^{n} F_{k l} A_{k}(x) B_{l}(y)
$$

where $O$ is the reconstructed image. According to formula (8), the computation takes particular component $F_{k l}$ and spread it to the appropriate region of $O$ with respect to $A_{k}$ and $B_{l}$. For details see [10].

In order to omit the damaged pixel from the computation, mask $S$ is used as follows

$$
F_{k l}=\frac{\sum_{y=0}^{N} \sum_{x=0}^{M} I(x, y) A_{k}(x) B_{l}(y) S(x, y)}{\sum_{y=0}^{N} \sum_{x=0}^{M} A_{k}(x) B_{l}(y) S(x, y)} .
$$

The mask is binary image where 0 denotes damaged pixel. According to formula (9), these pixels are not taken into consideration during the F-transform component computation.

This algorithm works well for photos [12, 13, 10, 14] but not so sufficiently for low depth color images. The reason is in isotropic nature of the algorithm where different regions are mixed together in the inpainted area $\Omega$. The addressed issue is illustrated in Fig. 4 .

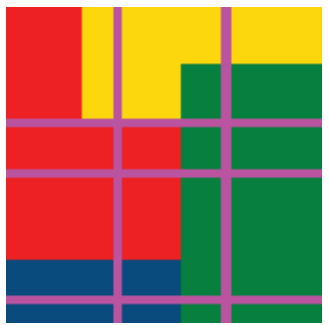

(a)

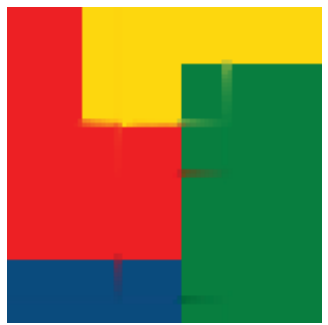

(b)

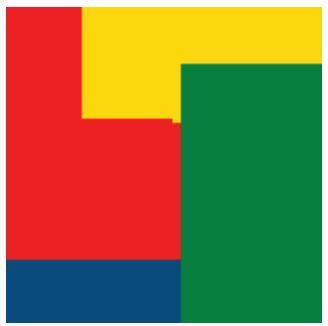

(c)

Figure 4: Demonstration of the unwanted blurriness in the reconstruction . a) Damaged image $I$; b) image inpainting using diffusion [10]; c) image inpainting using the proposed algorithm.

F-transform has been proven to work on various image processing tasks such as edge detection $[15,16]$, image fusion [17] or image compression [18]. 


\subsection{Fuzzy clustering}

Segmentation is important part of our technique. We propose to use fuzzy C-Means (FCM) which proved itself as very useful for image processing [19]. The algorithm was developed by J.C. Dunn [20] and improved by J.C. Bezdek [21]. It belongs to soft clustering which refers to the fact that each data point (pixel) belongs to the more than one cluster.

The input for the algorithm is set of elements $X=$ $\left\{\mathbf{x}_{1}, \ldots, \mathbf{x}_{n}\right\}$ into a collection of $K$ fuzzy clusters defined by centers $C=\left\{\mathbf{c}_{1}, \ldots, \mathbf{c}_{K}\right\}$ such as

$$
c_{k}=\frac{\sum_{x} w_{k}(x)^{m} x}{\sum_{x} w_{k}(x)^{m}},
$$

where $w_{k}(x)$ stands for membership degree of element $x$ to cluster $c_{k}$. Besides the clusters, the algorithm returns partition matrix $W=w_{i, j} \in[0,1], i=1, \ldots, n, j=$ $1, \ldots, K$ where $w_{i j}$ stands for membership degree of $x_{i}$ to cluster $c_{j}$.

The FCM minimize function

$$
\underset{C}{\arg \min } \sum_{i=1}^{n} \sum_{j=1}^{c} w_{i j}^{m}\left\|\mathbf{x}_{i}-\mathbf{c}_{j}\right\|^{2},
$$

where

$$
w_{i j}=\frac{1}{\sum_{k=1}^{c}\left(\frac{\left\|\mathbf{x}_{i}-\mathbf{c}_{j}\right\|}{\left\|\mathbf{x}_{i}-\mathbf{c}_{k}\right\|}\right)^{\frac{2}{m-1}}} .
$$

\section{NOVEL APPROACH DESCRIPTION}

We propose to divide an image to the several independent parts and process them one by one where the processing consists in image inpainting. The division is performed using fuzzy C-Means algorithm. Let us define discrete binary image $V_{i}:[0, M]_{Z} \times[0, N]_{Z} \rightarrow 0,1$ where the image is identified with cluster $c_{i}$ and pixels which belongs to it with maximum membership degree. All regions are inpainted using F-transform based algorithm and in the end, all of them are put together to create output image $O$.

\subsection{Algorithm}

Let us describe the algorithm on the image in Fig. 5.

First problem to solve is to determine to which cluster which damaged pixel belongs. For the rough estimation, we propose to use fuzzy image inpainting. Result is in Fig. 6.

In this step, the pixels from damaged region $\Omega$ are replaced by the blended colors of the surrounding ones.

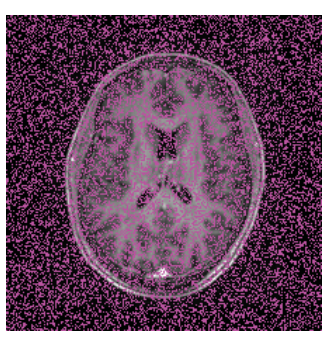

(a) $I$

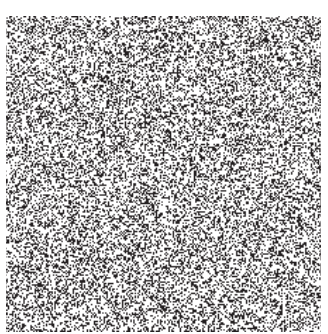

(b) $S$
Figure 5: a) Image $I$; b) mask $S$.

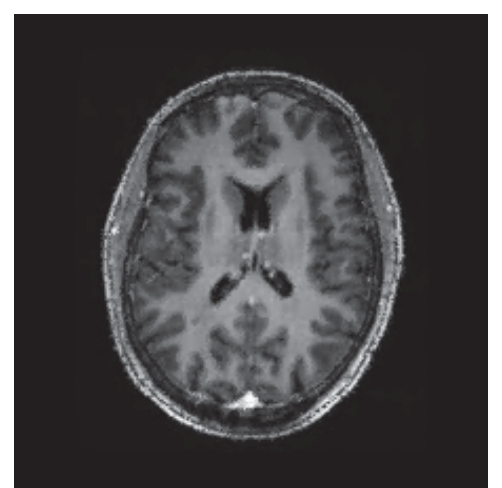

Figure 6: Image inpainting [10] of Fig. 5.

For small holes, the average of surrounding colors can be used. For bigger holes, it is better to use more advanced techniques. Because of a robustness, accessibility and a way of processing, we propose to use Ftransform inpainting described above which can handle both.

We assume that inpainting fills in $\Omega$ using colors from the close neighborhood. Thus, we can estimate the region used later for the separated reconstruction.

Because of the nature of the low color depth images, each blurriness caused by reconstruction is highly visible. The blurriness is caused by mixing of colors from the clearly separated places. Thus, we propose the separation for future region-by-region processing. For that purpose, fuzzy C-Means is proposed as can be seen in Fig. 7. The inpainted image from previous step is used as an input.

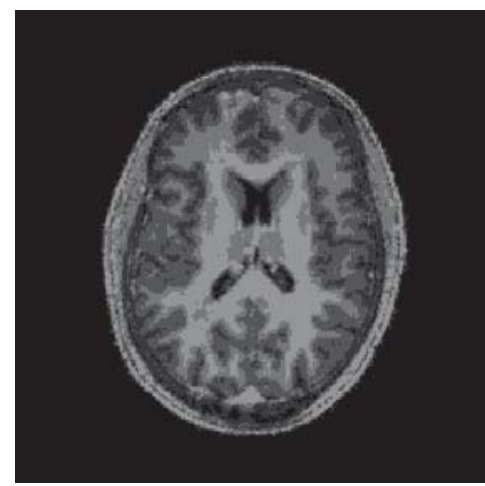

Figure 7: Fuzzy C-Means applied to Fig. 6. 
The regions are recognized using binary images $V_{1}, V_{2}, \ldots, V_{K}$ identified with the clusters. Image $V$ labels valid pixels. Therefore, the separated regions are reconstructed from pixels of the known part of themselves. This feature makes the inpainting algorithm anisotropic which prevents the unwanted color mixing. Demonstration of the segmented regions for $K=5$ is in Fig. 8.
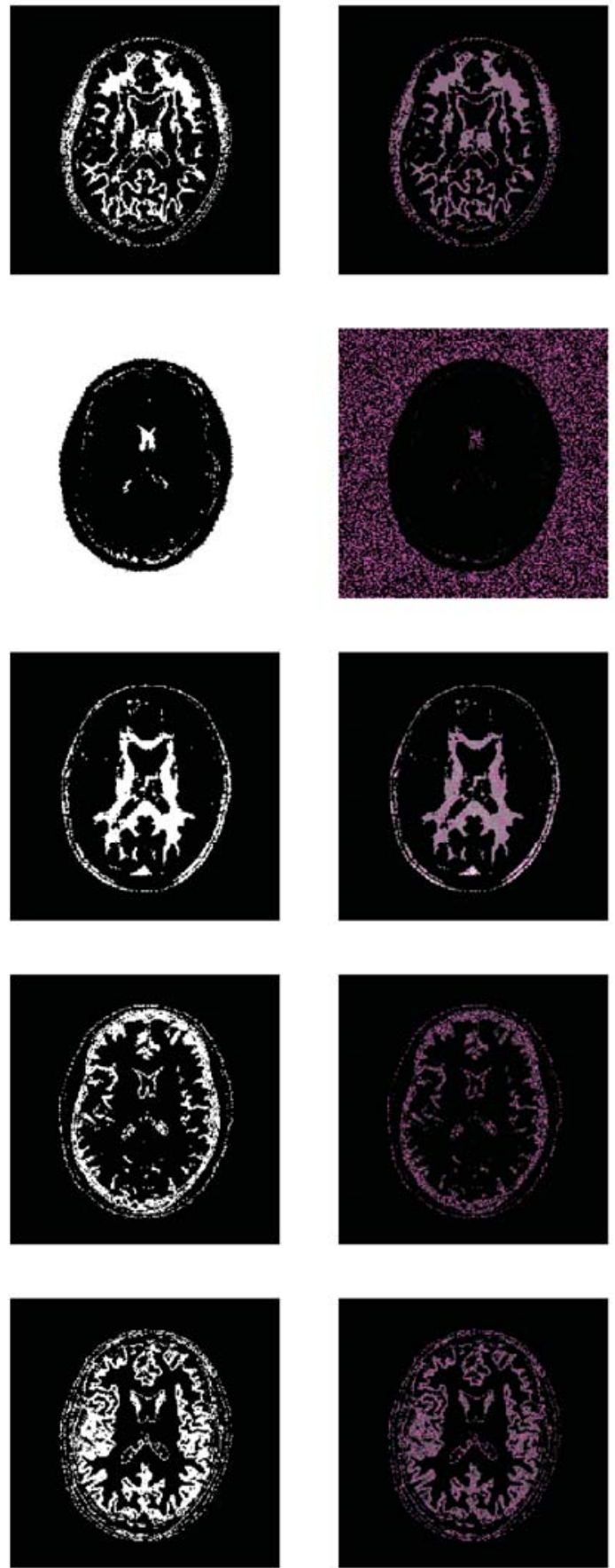

Figure 8: Left column contains images $V_{1}, V_{2}, V_{3}, V_{4}, V_{5}$ and right column respective parts of input image $I$. The division is based on Fig. 7.
Next step is to create a set of masks $S_{1}, S_{2}, \ldots, S_{K}$ to use together with $V_{1}, V_{2}, \ldots, V_{K}$. Each mask labels damaged pixels just in particular region. The image $V$ influences the computation of the F-transform components as follows

$$
F_{k l}=\frac{\sum_{y=0}^{N} \sum_{x=0}^{M} I(x, y) S(x, y) V(x, y) A_{k}(x) B_{l}(y)}{\sum_{y=0}^{N} \sum_{x=0}^{M} S(x, y) V(x, y) A_{k}(x) B_{l}(y)} .
$$

Each region is inpainted independently using proper $S$ and $V$. Example for $K=5$ is illustrated in Fig. 9. For better illustration, Fig. 10 shows detail of a brain and comparison between our novel algorithm and an original one.
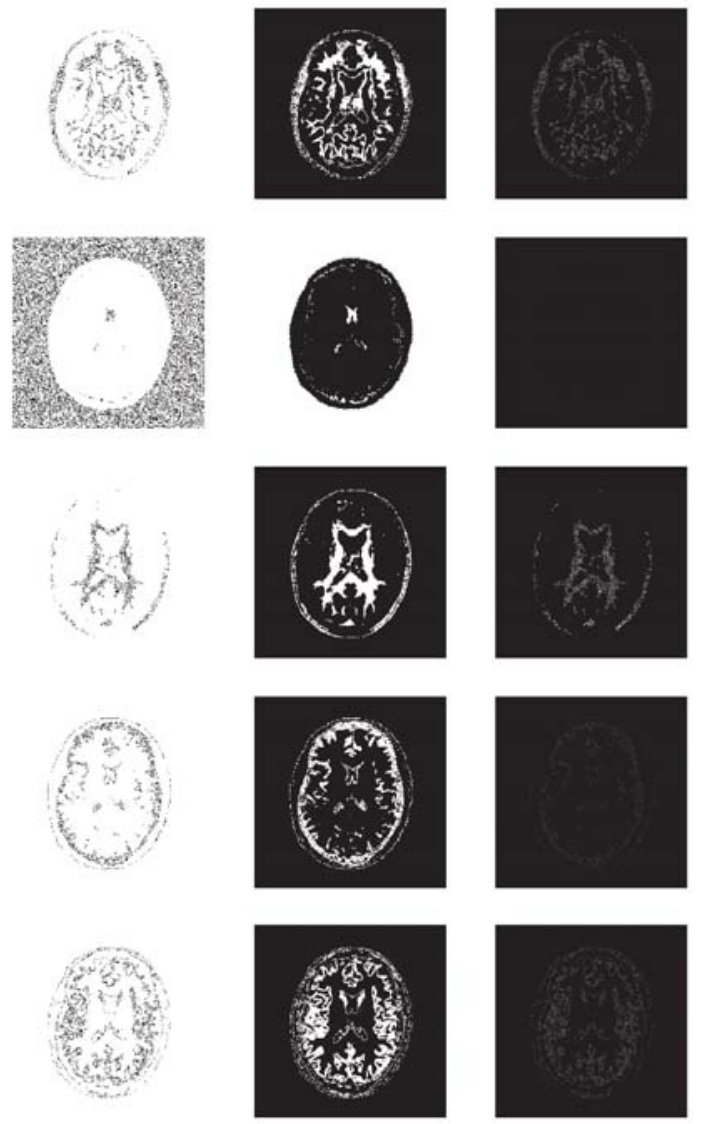

Figure 9: Left column contains masks $S_{1}, S_{2}, S_{3}, S_{4}, S_{5}$. Middle one contains valid pixels $V_{1}, V_{2}, V_{3}, V_{4}, V_{5}$ and right one reconstructed parts in each region. The division is based on Fig. 7 .

The proposed algorithm is focused on the specific images which are very sensitive to blurred reconstruction due to their low depth colors. Thus, the algorithm is demonstrated on medical images in Fig. 11 and applied to each color channel.

Let us summarize all steps:

1. Inpaint input image using conventional F-transform image inpainting. 


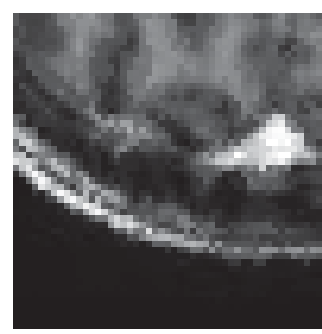

(a)

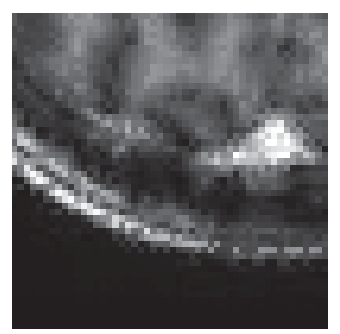

(b)

Figure 10: a) Novel algorithm with more clear and unified output; b) original algorithm [10]. Adjacent colors are more uniform for figure a). Visible difference is in white spot region and even not so shattered edge.

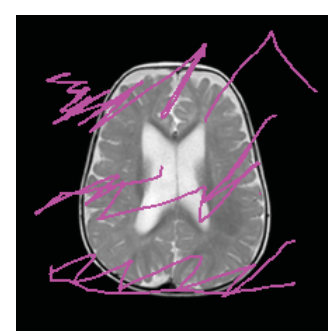

(a) Brain

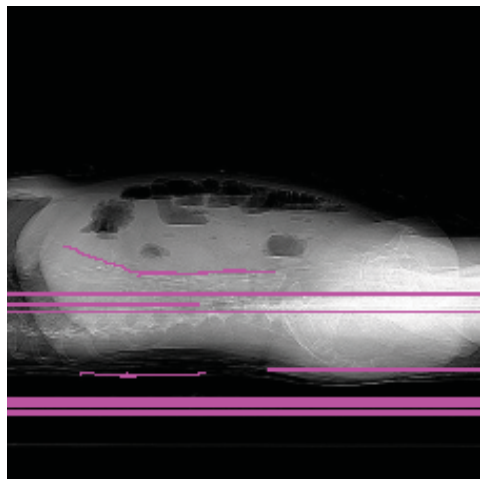

(c) Torso

Figure 11: A set of images for demonstration purposes.

2. Cluster the inpainted image using fuzzy C-Means.

3. Identify each cluster region with binary image $V_{i}$.

4. Separate input mask $S$ to the several regions $S_{i}$ based on clusters.

5. Inpaint input image region by region using appropriate $V_{i}$ and $S_{i}$.

Implementation of our new technique is based on publicly available F-transform source code from OpenC $\mathrm{V}^{2}$. For testing images, one reconstruction lasts few seconds on the average PC. Results are available in Fig. 12.

A deep comparison with plenty of techniques is not possible due to lack of their implementations and/or their

\footnotetext{
${ }^{2}$ opencv-contrib framework, module fuzzy
}

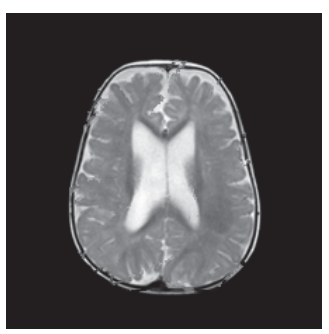

(a) Brain

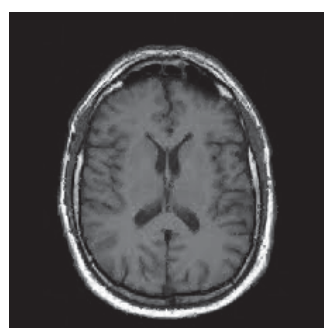

(b) Brain

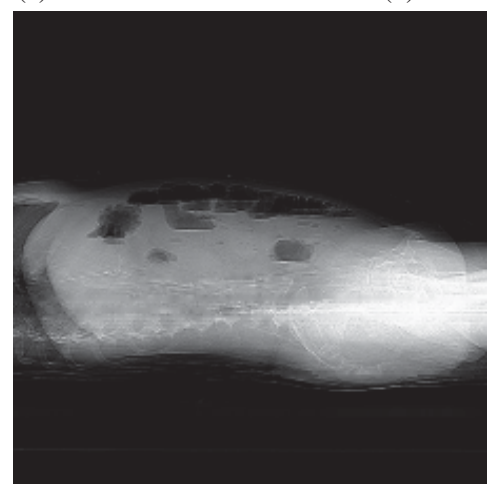

(c) Torso

Figure 12: Image inpainting of Fig. 11. Target was to erase purple text, lines and noise.

inabilities to work with specific types such as noisy images $^{3}$.

\subsection{Qualitative comparison}

Let us compare the novel technique with basic principle of the enhanced diffusion idea [10], Navier-Stokes [8] and FMM [9]. The various outputs can be seen in Fig. 13, 14 and 15.

Due to size limitation, the details are not obvious. Let us demonstrate the main feature of the proposed technique which is to keep sharp reconstruction without blurred artifacts. The details are given in Fig. 16.

\section{CONCLUSION}

In this initial study, we proposed a technique aimed to low color depth images usable for medical ones from MRI or CT. Its novelty consists in separation according to colors followed by region-by-region processing. For the separation, the fuzzy C-Means is proposed and for the processing the fuzzy image inpainting. To make the separation possible, we need to estimate to what clusters all damaged pixels belong. For that purpose, it is necessary to do inpainting of the whole image as a first step.

Disadvantage of the common techniques lays in their isotropic way of processing. As was demonstrated, this

\footnotetext{
${ }^{3}$ For instance, the exemplar based techniques are not effective for highly damaged pictures, moreover with damage distributed all over them.
} 


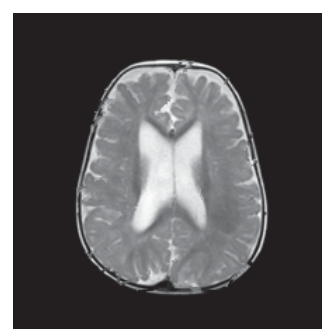

(a)

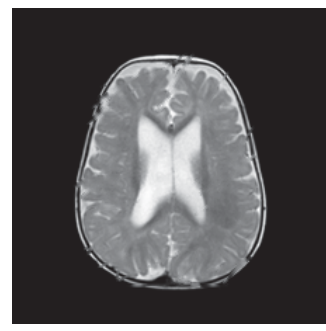

(c)

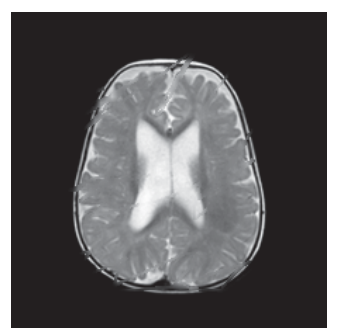

(b)

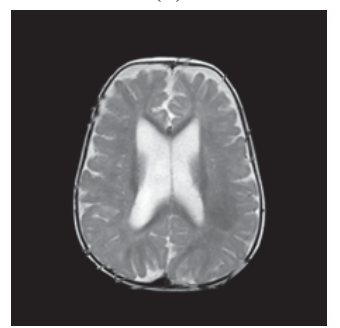

(d)
Figure 13: Comparison of the various image inpainting techniques. a) Novel algorithm; b) fuzzy image inpaintg; c) Navier-Stokes; d) FMM.

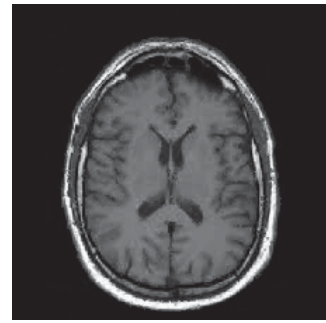

(a)

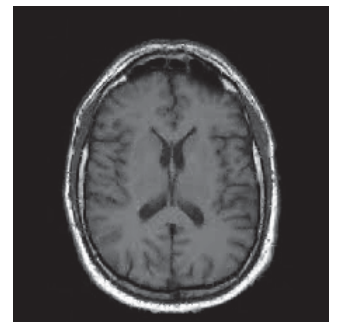

(c)

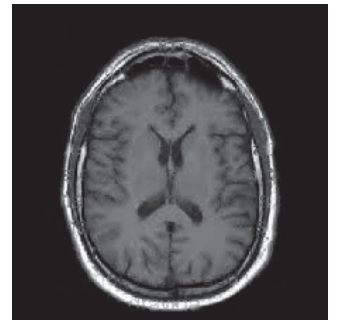

(b)

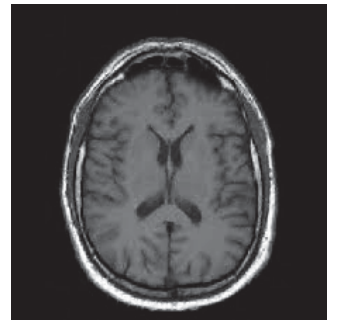

(d)
Figure 14: Comparison of the various image inpainting techniques. a) Novel algorithm; b) fuzzy image inpaintg; c) Navier-Stokes; d) FMM.

fact leads to blurry reconstruction which is more obvious in low depth color images. The separation part of our algorithm successfully prevents this issue and in fact it could come after any common inpainting algorithm as a second step. To achieve this, we use submask labeling the damaged pixels of the particular region and valid pixels labeling pixels of the cluster dedicated to the same region. User should provide image, mask and specify a number of clusters. Everything else is performed automatically by algorithm and the implementation respectively.

We explained the way of working of our algorithm and compared it with another techniques. For future re-

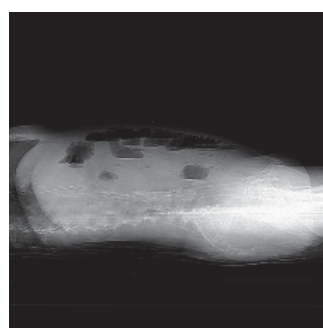

(a)

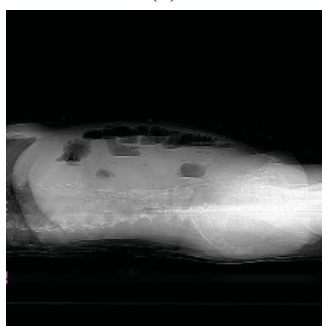

(c)

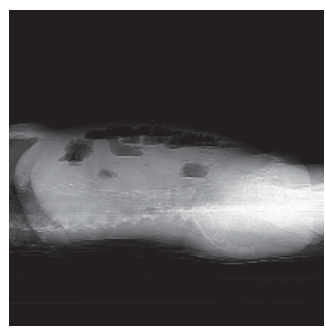

(b)

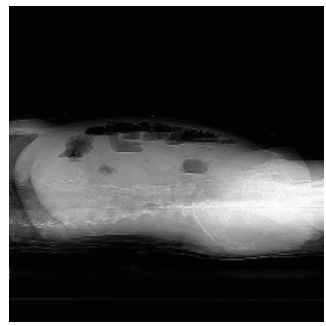

(d)
Figure 15: Comparison of the various image inpainting techniques. a) Novel algorithm; b) fuzzy image inpaintg; c) Navier-Stokes; d) FMM.

search, we would like to extend the idea for heuristic and probability estimation of the reconstructed part specifically aimed to medical usage.

\section{ACKNOWLEDGMENT}

This work was supported by The Ministry of Education, Youth and Sports from the National Programme of Sustainability (NPU II) project "IT4Innovations excellence in science - LQ1602".

\section{REFERENCES}

[1] M. Bertalmio, G. Sapiro, V. Caselles, and C. Ballester, "Image inpainting," in Proceedings of the 27th annual conference on Computer graphics and interactive techniques. ACM Press/Addison-Wesley Publishing Co., 2000, pp. 417-424.

[2] A. A. Efros and T. K. Leung, "Texture synthesis by non-parametric sampling," in Computer Vision, 1999. The Proceedings of the Seventh IEEE International Conference on, vol. 2. IEEE, 1999, pp. 1033-1038.

[3] A. Criminisi, P. Pérez, and K. Toyama, "Region filling and object removal by exemplar-based image inpainting," Image Processing, IEEE Transactions on, vol. 13, no. 9, pp. 1200-1212, 2004.

[4] N. Komodakis and G. Tziritas, "Image completion using efficient belief propagation via priority scheduling and dynamic pruning," Image Processing, IEEE Transactions on, vol. 16, no. 11, pp. 2649-2661, 2007.

[5] I. Drori, D. Cohen-Or, and H. Yeshurun, "Fragment-based image completion," in ACM 
Transactions on Graphics (TOG), vol. 22, no. 3. ACM, 2003, pp. 303-312.

[6] J. M. Ogden, E. H. Adelson, J. R. Bergen, and P. J. Burt, "Pyramid-based computer graphics," RCA Engineer, vol. 30, no. 5, pp. 4-15, 1985.

[7] M. Elad, J.-L. Starck, P. Querre, and D. L. Donoho, "Simultaneous cartoon and texture image inpainting using morphological component analysis (mca)," Applied and Computational Harmonic Analysis, vol. 19, no. 3, pp. 340-358, 2005.

[8] M. Bertalmio, A. L. Bertozzi, and G. Sapiro, "Navier-stokes, fluid dynamics, and image and video inpainting," in Computer Vision and Pattern Recognition, 2001. CVPR 2001. Proceedings of the 2001 IEEE Computer Society Conference on, vol. 1. IEEE, 2001, pp. I-355.

[9] A. Telea, "An image inpainting technique based on the fast marching method," Journal of graphics tools, vol. 9, no. 1, pp. 23-34, 2004.

[10] I. Perfilieva and P. Vlašánek, "Image reconstruction by means of F-transform," Knowledge-Based Systems, vol. 70, pp. 55-63, 2014.

[11] I. Perfilieva, "Fuzzy transforms: Theory and applications," Fuzzy sets and systems, vol. 157, no. 8, pp. 993-1023, 2006.

[12] I. Perfiljeva, P. Vlašánek, and M. Wrublova, "Fuzzy transform for image reconstruction," Uncertainty Modeling in Knowledge Engineering and Decision Making, pp. 615-620, 2012.

[13] P. Vlašánek and I. Perfilieva, "Image reconstruction with usage of the F-transform," in International Joint Conference CISIS'12-ICEUTEÂ'12SOCOÂ'12. Springer, 2013, pp. 507-514.

[14] _ , "Interpolation techniques versus Ftransform in application to image reconstruction," in 2014 IEEE International Conference on Fuzzy Systems (FUZZ-IEEE). IEEE, 2014, pp. 533539.

[15] I. Perfilieva, P. Hodáková, and P. Hurtík, "Differentiation by the F-transform and application to edge detection," Fuzzy Sets and Systems, 2014.

[16] M. Danková, P. Hodáková, I. Perfilieva, and M. Vajgl, "Edge detection using F-transform." in ISDA, 2011, pp. 672-677.

[17] M. Vajgl, I. Perfilieva, and P. Hod'áková, “Advanced F-transform-based image fusion," $A d$ vances in Fuzzy Systems, vol. 2012, p. 4, 2012.

[18] I. Perfilieva and B. De Baets, "Fuzzy transforms of monotone functions with application to image compression," Information Sciences, vol. 180, no. 17, pp. 3304-3315, 2010.

[19] M. J. Christ and R. Parvathi, "Fuzzy c-means algorithm for medical image segmentation," in
Electronics Computer Technology (ICECT), 2011 3 rd International Conference on, vol. 4. IEEE, 2011, pp. 33-36.

[20] J. C. Dunn, "A fuzzy relative of the isodata process and its use in detecting compact wellseparated clusters," Cybernetics, vol. 3, pp. 3257, 1973.

[21] J. C. Bezdek, "Objective function clustering," in Pattern recognition with fuzzy objective function algorithms. Springer, 1981, pp. 43-93. 


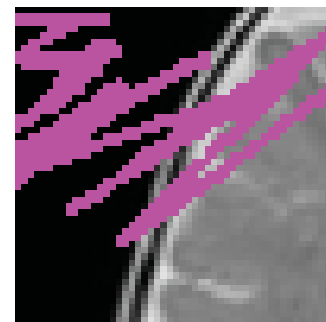

(a)

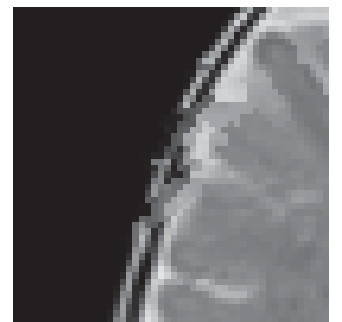

(b)

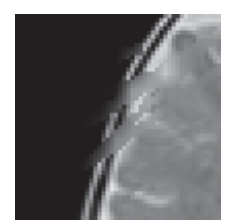

(c)

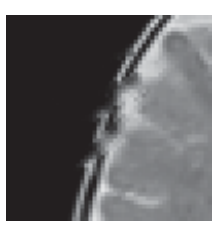

(d)

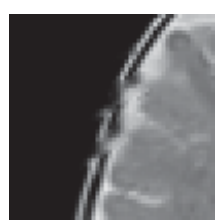

(e)

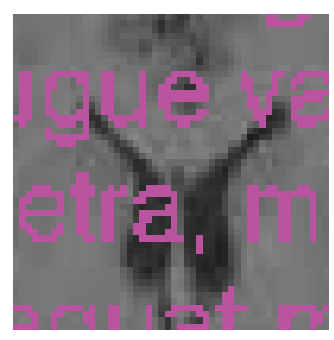

(f)

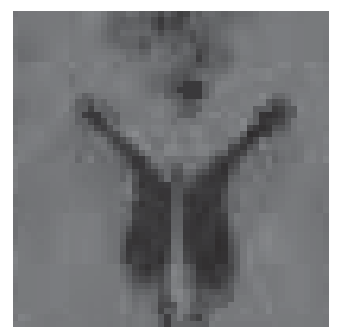

(g)

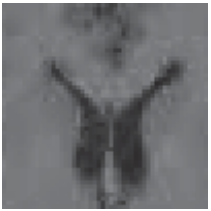

(h)

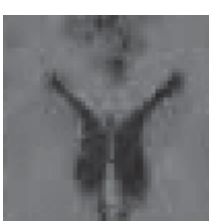

(i)

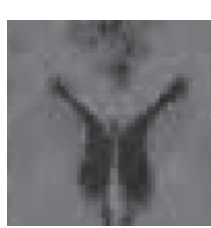

(j)

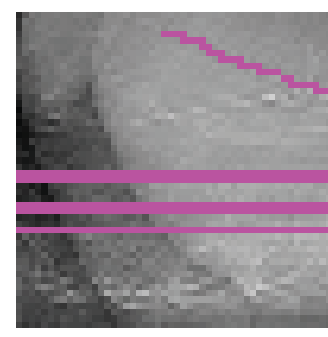

(k)

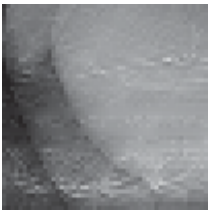

(m)

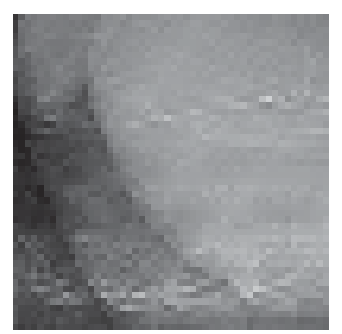

(1)

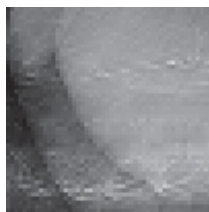

(o)

Figure 16: Details of the reconstruction in Fig. 15, 13 and 14. a) f) k) Damaged image; b) g) l) image inpainted by the novel algorithm; c) h) m) image inpainted by original fuzzy inpainting [10]; d) i) n) image inpainted by Navier-Stokes [8]; e) j) o) image inpainted by FMM [9]. 